\title{
Young adult renal service: Does having a dedicated young adult service make difference to patient outcomes?
}

\section{Samantha Williamson}

University Hospitals Birmingham NHS Foundation Trust

Jemma Myton

University Hospitals Birmingham NHS Foundation Trust

Felicity Evison

University Hospitals Birmingham NHS Foundation Trust

\section{Larissa Kerecuk}

Birmingham Women's and Children's NHS Foundation Trust

\section{Graham Lipkin}

University Hospitals Birmingham NHS Foundation Trust

\section{Tanya Pankhurst ( $\triangle$ tanya.pankhurst@uhb.nhs.uk)}

University Hospitals Birmingham https://orcid.org/0000-0002-8341-668X

\section{Research article}

Keywords: ,

Posted Date: October 15th, 2019

DOI: https://doi.org/10.21203/rs.2.16057/v1

License: (1) This work is licensed under a Creative Commons Attribution 4.0 International License. Read Full License 


\section{Abstract}

Background Young adults with chronic renal disease face the rigours of adolescence alongside the responsibility of safeguarding their own health. They have poorer renal transplant outcomes than other age groups presumed due to non-adherence with medication and clinic attendance. We had set up a Young Adult Service and aimed to understand whether this had reduced distress in young adults and impacted positively on renal transplant survival.

Methods Transplant patients aged 16-24 years who had transferred to adult renal services between 2000 and 2006, prior to commencement of a dedicated young adult service, were studied as historical controls. This patient group was compared to the current young adult transplant cohort. Missed appointments, serial renal function and transplant survival was collected. The current young adult cohort were assessed for markers of 'distress' and this was correlated with clinic attendance and transplant outcomes. Young adults were surveyed for their opinion of the service.

Results Five year transplant survival increased from $65 \%$ to $98 \%$ concurrent with Young Adult Service commencement. $60 \%$ of patients reported distress and this correlated with missed clinic appointments. By offering more appointments to this group and increasing support, neither distress nor missed appointments impacted transplant outcomes despite the high risk in this patient cohort. The service was well received by young adults.

Conclusion A dedicated Young Adult Renal Service may protect from poor renal transplant outcomes by providing increased holistic support.

\section{Background}

Young adults in the UK with kidney transplants lose their grafts more frequently than in any other age group (unpublished UK data, submitted for publication) and young adults report disorientation and a feeling of loss as they move from paediatric to adult care (1). These problems have created interest within the NHS: the Department of Health (2), National Institute for Clinical Excellence (NICE) (3) and the Care Quality Commission (CQC) (4) have published guidance on providing a tailored, patient-centred service, led by a multi-disciplinary team. Typically a young adult team involves both paediatric and adult clinicians, youth workers and specialist nurses, and aims to offer holistic care and support to both the patient and their carers during transition from paediatric to adult care (5).

Young adult services in renal medicine have been developed to varying degrees nationally but the evidence surrounding whether such services are successful, either impacting health outcomes, or improving well-being as reported by young adults themselves, is limited. In the UK, Harden et al (6) studied a small patient cohort study reporting no loss of grafts in a patient group with a dedicated young adult service, compared to a graft loss of $67 \%$ in the pre-transitional historical control group. The 'Ready, Steady, Go' transitional programme (7) is being increasing adopted by many NHS trusts and is well received although has not reported outcome improvement. Internationally, the Zurich transitional care 
programme has shown a significantly lower rate of decline in eGRF and reduction in acute rejection episodes of transplanted adolescents under transitional care (8). A Canadian study reported nonrecordable calcineurin levels dropping from $63 \%$ to $19 \%(p=0.03)$ after the initiation of a young adult service (9).

University Hospitals Birmingham NHS Foundation Trust (UHB), along with Birmingham Women's and Children's NHS Foundation Trust (BWC) has a well-established Young Adult Renal Service which was set up in 2006 to provide care for young adult renal patients between the ages of 16 and 25 years.

We were interested in understanding whether the Young Adult Service had impacted on renal transplant outcomes when compared to a historical non-transitioned cohort; whether there were any patients at particular risk of poor transplant outcomes (including the proportion of current patients reported psychological distress) and the feedback from young adults themselves.

\section{Methods}

A mixed methods model was used in this study, with quantitative comparisons and qualitative surveys.

\section{Patient Groups}

Two patient groups were included in this study. Transplant patients aged 16-24 years of age who had transferred to UHB Renal Service between 2000 and 2006, prior to commencement of a dedicated Young Adult Service were studied as historical controls. The second patient group were patients in the young adult service at the time of the study with a functioning transplant.

\section{Data Collection tools and methods}

Electronic Health Records (EHR) in our hospital are ubiquitous and allow for extraction of clinical data from routine clinic appointments. Prior to this study, the Young Adult Service had commissioned the development of a specialised database to allow a standardised, coordinated collection of psychosocial data from young adults for clinical use, in order to highlight patients at risk within the clinic.

Information for this database was collected via a scripted consultation, over the telephone or in person at routine clinic appointments. At the initiation of this database, patients were informed prior to the consultation of the nature of the project, and that they would be contacted to provide baseline information with the intention to gather ongoing data throughout their follow up in the Young Adult Service. This would identify care issues; improving quality of care both for individual patients and for the service as a whole. Patients were informed that data would be held securely within the hospital EHR and would be audited anonymously for service improvement. Individual data would be discussed with and added to patient records throughout their patient journey. Patients were also informed that they did not have to participate in answering any questions they did not want to, as with standard medical 
consultations. A standardised second questionnaire was developed to ask users to assess the service. This was distributed at clinics on paper and returned anonymously via clinic support staff (appendix 1).

When patients were unavailable by telephone or did not attend (DNA) appointments, they were contacted as our standard practice to follow up patients who do not attend.

Data was extracted by the Informatics Department complying with Caldicott Guardianship rules, the data were anonymised before release to investigators. The study was registered with the Clinical Audit Department (CARMS number 13375).

\section{Data Collected}

Routine clinical information and laboratory results were available from clinic consultations including trends in serial serum creatinine and urine albumin to creatinine ratios, rates of return to dialysis, retransplantation, clinic attendance and 5- and 10-year graft survival. Diagnoses and whether the patient was transitioned from paediatric care or presented directly to adult services were extracted. Clinic attendance and the number of clinics patients did not attend were recorded. In the young adult service, if an appointment is not attended, another one is automatically offered in the next available clinic; this data was also collected.

Additional data collected at baseline or in routine appointments included diagnosis of additional learning needs (including attention deficient-hyperactivity disorder, autism and developmental problems) and prevalence of symptoms associated with renal disease. A scripted psycho-social questionnaire (HEADSS (Home, Education and employment, Activities, Drugs, Sexuality, and Suicide and Depression) already in use in adolescent services (10) to assess broad issues affecting young adults, was performed, collecting current information on home life, diet, employment, mood, suicidal ideation, drug/ alcohol use, medication adherence and relationships. Participants were scored on their responses using pre-assigned definitions (Table 1). For example for the domain 'Home' a judgement by the interviewer was made as to whether responses corresponded with a 'supportive home life,' 'conflicts at home' or 'dysfunctional home life'. Interviews were performed by a single member of staff to limit variation in scoring. If the young adult was unable to take part in the interview due to learning difficulties or communication difficulties, the interview was conducted with a parent or guardian. 'Distress' was recorded if response scoring of the HEADDS questionnaire reported evidence of unhappiness/conflict.

Descriptive analyses were used to make comparisons between the demographics of the current young adult transplant cohort and the historical cohort. Categorical variables were presented as numbers and percentages and compared using chi-squared tests. Continuous variables were presented as either mean or median with comparisons made using Mann-Whitney tests for medians and t-test for means. Survival analyses were performed by generation of Kaplan-Meier curve estimates and compared between groups using log-rank tests. Survival rates at 5 and 10 years were calculated using life tables. To evaluate whether there was an association between decline in estimated glomerular filtration rate (eGFR) and 
learning difficulties, distress or DNA rates, a linear regression model was created for each patient, given the assumption that eGFR and time had a linear relationship, to calculate a slope. This slope was then used to estimate each patient's percentage change in eGFR over time, dividing the slope by the baseline value to calculate the annual eGFR decline.

A multivariable linear mixed effects model was created, with fixed and random intercept and slope, to allow for unequally spaced time points and an uneven number of measurements per patient. Anonymised patient identifier was added as a random effect to represent variability between a patient's eGFR trajectory and to account for correlation between measurements of the same patient, taking into account the fact that each patient can have multiple measurements within the dataset. All analysis was conducted using Stata SE 14 (Stata Statistical Software: College Station, TX: Stata Corp LP)(11). Pvalues $<0.05$ were considered significant.

\section{Results}

\section{Demographics of patient groups (Table 1)}

The current young adult transplant cohort consisted of 71 patients with $54 \%$ males. Patients in this cohort were first seen at the adult centre at the median age of 16 years. The age of patients ranged from 16-27 (mean 20 years). Due to the tertiary status of both our paediatric and adult centres, a large number of renal related diagnoses were seen. The cohort was mostly transferred from a paediatric centre (96\%) and underwent a formal transition programme, with $4 \%$ of patients newly diagnosed patients presenting to the adult service.

The historical transplant cohort consisted of 48 patients, who had entered the adult services prior to the introduction of the dedicated Young Adult Service. The cohort consisted of $46 \%$ males. Introduction to the adult service was at an older age (19 years) that the current young adult transplant cohort.

\section{Outcome Data}

Data for patients in both groups was taken from their transplant date to their most recent appointment up to April 2017. Transplant failure was defined as re-transplant, return to dialysis or death.

As expected the historic cohort had longer median follow up (18 years) compared to the current cohort (8 years).

Of the historical cohort, 35 out of 48 (71\%) experienced transplant failure within their follow up time. 5year graft survival was $65 \%(\mathrm{Cl} 49-76 \%)$ and 10-year graft survival rates were 50\% $(\mathrm{Cl} 35-63 \%)$.

Of the current young adult cohort, 11 out of the 71 transplant patients (15\%) experienced graft failure within their follow up time. The 5-year graft survival was $98 \%(\mathrm{Cl} 88-99 \%)$ and the 10 -year graft survival was $81 \%(\mathrm{Cl} 64-91 \%)$. 


\section{Risk factors for Renal Graft Failure: Young Adult Renal Transplant Cohort}

\section{'Distress' related to transplant failure}

In the young adult transplant cohort, 43 patients (60\%) reported distress during the psychosocial questionnaire.

There was no difference in the baseline eGFR between patients who recorded distress or no distress, respectively (74 $\mu \mathrm{mol} / \mathrm{L}$ (SD 27.33), $83 \mu \mathrm{mol} / \mathrm{L}$ (SD 10.86), $p=N S$ ). There was no difference in the annual average renal function decline rate for patients with distress and no distress $(-0.22 v-0.08, p=N S)$. In multivariable mixed regression, distress was not significant. The proportion of transplant patients who had reported distress and no distress and subsequently lost their transplant was 18\% $(n=6)$ and 15\% (n $=4$ ), respectively and this was not statistically significant.

\section{Learning difficulties}

Of the young adult transplant cohort, 22 patients $(31 \%)$ were reported to have some degree of learning difficulties. Learning difficulties did not impact renal transplant loss. Of those with a recorded learning difficulty, $18 \%$ experienced a failure event, compared with $14 \%$ of those with no learning difficulties $(p=$ NS).

\section{Missed Appointments}

On average, patients were offered 4.1 appointments at the Young Adult Clinic per year, and on average, young adults did not attend 1.2 of appointments offered per year.

There was no difference in the proportion of missed appointments in patients who did or did not experience graft failure: $13 \%(82 / 736)$ of appointments in patients with graft loss were missed and $13 \%$ $(243 / 1845)$ of appointments in those who did not lose graft were missed.

In the young adult clinic, patients who did not attend are contacted and offered another appointment in the next clinic. More appointments were, therefore, offered to those patients who regularly miss appointments. Patients who missed fewer than 3 appointments per year were offered an average of 4.7 appointments per year. Patients who missed more than 3 appointments per year were offered an average of 10.3 appointments per year. There was no significant difference in the failed transplant rates related to the number of appointments offered (mean 3.59 vs. mean $5.21 \mathrm{p}=\mathrm{NS}$ ). Absolute number of missed appointments was also not significant.

Patients who reported distress were offered an average of 7.68 appointments per year. Patients who reported no distress were offered an average of 2.26 appointments per year $(p<0.001$.) Patients reporting 
distress did not miss more appointments than patients reporting no distress (12\% versus $15 \%$ ( $p=$ 0.075)).

\section{Service Evaluation}

In the questionnaire directly evaluating the service users reported that they were satisfied with the service. In a survey of 71 users there were 39 responses (Table 2). Users reported satisfaction with the service and reported it helpful in understanding and managing their disease and medications. $64 \%$ of users thought that text message reminders for clinics would be helpful, very few wanted more group support (13\%) or peer support (10\%). There was little demand for clinic appointments outside the hospital setting $(5 \%)$.

\section{Discussion}

The paediatric management of long term illness often differs from the traditional adult based model, with a more paternalistic approach applied with prominent parental involvement. Provision of a personalised service has been linked to improved patient outcomes $(6,9)$. Transition has been defined as the 'purposeful, planned process that addresses the medical, psychological and educational/vocational needs of adolescents and young adults' (1).

A dedicated Young Adult Renal Service has been available in Birmingham since 2006, comprising of adult and paediatric nephrologists, dedicated paediatric and adult nurses, a youth worker, pharmacist and psychologist; and offers longer appointment times, support outside the hospital and inpatient support. Patients are now introduced to the adult team earlier when compared historical cohort.

Five and ten-year renal transplant survival was shown to be significantly better in the transitioned cohort when compared with the historical cohort, with significant reduction in graft failure. This could be partly attributed to the introduction of a dedicated Young Adult Service. However, other factors need to be taken into account. Using a historical cohort as the comparator cohort means the cohorts cannot be matched. The standard of medical care, process of transplantation, choice of donors and immunosuppressant drugs used routinely have changed, and the improved outcomes are likely to be multifactorial. However, the overall improvement in 5-year transplant survival in the UK over this time period has only increased by $2 \%$ (12) which encouragingly suggests that the Young Adult Service may have impacted survival rates which improved from $65 \%$ in historical cohort to $98 \%$ in the Young Adult Clinic cohort.

The burden of chronic kidney disease and the process of transplantation is heavy, especially on an adolescent who also faces many other challenges. Current service users reported 'distress' in key life domains such as relationships, employment and mood when assessed with the HEADDSS questionnaire. We believe it is important to offer holistic care and multi-faceted support, which is easier to provide in a multidisciplinary service than a traditional clinic set-up. In the current patient cohort, $60 \%$ of patients reported distress. No significant difference in decline of eGFR or graft failure was seen when compared to those with no positive markers of distress. Importantly, patients who have evidence of distress were 
offered, and attended, significantly more clinic appointments than those that reported no distress. The clinic is supporting these patients and we postulate that this is protective. Qualitative data in the form of a feedback questionnaire was positive, with patients feeling the service enables them to manage their own illness more independently and prepares them for adult life.

The current cohort did not attend $13 \%$ of their appointments. Not attending clinic appointments has been shown to have a significant effect on HbA1c in young adult diabetes clinics (13). We did not find missed appointment rates were higher in patients who lost their transplants or in patients who reported distress on questioning. There is an active support structure within the clinic to try and prevent non-attendance: the Youth Worker and Specialist Nurses actively engage young adults before the clinic; especially if they have missed previous appointments.

Adolescents have been shown to have the highest rates of acute renal transplant rejection and rejection related graft loss of any age group. National small scale studies have demonstrated that adolescence is a critical time period related to graft survival with one centre reporting $35 \%$ of their adolescent transplants failed unexpectedly within 36 months of transfer (14). Internationally, it has been demonstrated that adolescent transplant patients have poorer outcomes compared to younger children and older adults, with recipients of renal transplants at adolescence demonstrating the greatest risk of graft failure in the USA $(15,16)$. This trend has been related to poor medication adherence; with meta-analysis demonstrating $32 \%$ of adolescent kidney transplant recipients were non-adherent (17). Co-existing learning difficulties, autism and developmental delay are seen in multi-system rare disease which can include renal impairment, and these needs require further alteration to the service model. In these situations, traditional aims of empowering independence and education are often superseded by the need to support parents in caring for a young adult, where access to Social Care becomes problematic due to the cessation of childhood services (18). In patients who were distressed we found higher rates of nonattendance at clinic but we were able to counter this by offering more appointments and encouraging attendance.

Further investigation into effectiveness of young adult services will be possible as more data becomes available, especially as digital records become more ubiquitous. Analysis of suspected high risk groups, such as those with depression, anxiety, unemployment or relationship issues are likely to be most in need of supportive intervention.

\section{Conclusions}

We postulate that renal transplant graft survival rates are positively impacted by a Young Adult Service. The high rate of non-attendance traditionally seen in young adult clinics can be addressed by increasing clinic appointments offered and by provision of a dedicated multidisciplinary team with expertise and dedication to adolescents and young adults. Distress is high in young adults and compounded by medical problems. Patients who are at high risk of graft failure may be protected from poor outcomes by dedicated services. 


\section{Abbreviations}

eGFR-estimated Glomerular Filtration Rate

Cl- confidence intervals

HEADDS questionnaire - Home, Education and employment, Activities, Drugs, Sexuality, and Suicide and Depression questionnaire

DNA-did not attend

EHR-Electronic Health Record

\section{Declarations}

\section{Ethics approval and consent to participate and Consent for publication}

All patients were verbally consented for collection of information for clinical use and service improvement. No identifiable information was passed to investigators during evaluation. The study was performed as an audit and therefore no ethics committee approval was necessary (19). No participants were under 16 years of age.

\section{Availability of data and materials}

Data are derived from the clinical records via the Informatics department of the institution and although anonymised are not available for onward sharing.

\section{Competing interests}

The authors declare they have no competing interests

\section{Funding}

This research did not receive any specific grant from funding agencies in the public, commercial, or notfor-profit sectors

\section{Authors' contributions}


SW, TP, LK and GL conceived the ideas for the study, wrote the paper and revised subsequent versions. JM and FE analysed the datasets. All authors have read and approved the manuscript.

\section{Acknowledgements}

We are grateful to administration staff who collected surveys and all service users who participated in providing feedback.

\section{References}

1.Blum RW, Garell D, Hodgman CH, Jorissen TW, Okinow NA, Orr DP, et al. Transition from child-centered to adult health-care systems for adolescents with chronic conditions. A position paper of the Society for Adolescent Medicine. J Adolesc Health. 1993;14(7):570-6.

2.Department of Health GoU. Quality criteria for young people friendly health services.

https://wwwgovuk/government/publications/quality-criteria-for-young-people-friendly-health-services. 2011.

3.NG43 Ng. Transition from children's to adults' services for young people using health or social care services NICE guideline [NG43]. 2016:https://www.nice.org.uk/guidance/ng43.

4.Commission CaQ. From the pond to the sea: Childrens transition to adult health services. 2014.

5.British Association for Paediatric Nephrology RA. Helping adolescents and young adults with end stage renal failure 2009:https://renal.org/wp-content/uploads/2017/06/RA_BAPN_-

_Helping_Adolescents_and_Young_Adults_with_ESRF_-_July_09.pdf.

6. Harden PN, Walsh G, Bandler N, Bradley S, Lonsdale D, Taylor J, et al. Bridging the gap: an integrated paediatric to adult clinical service for young adults with kidney failure. BMJ. 2012;344:e3718.

7.Nagra A, McGinnity PM, Davis N, Salmon AP. Implementing transition: Ready Steady Go. Arch Dis Child Educ Pract Ed. 2015;100(6):313-20.

8.Weitz M, Heeringa S, Neuhaus TJ, Fehr T, Laube GF. Standardized multilevel transition program: Does it affect renal transplant outcome? Pediatr Transplant. 2015;19(7):691-7.

9.McQuillan RF, Toulany A, Kaufman M, Schiff JR. Benefits of a transfer clinic in adolescent and young adult kidney transplant patients. Can J Kidney Health Dis. 2015;2:45.

10.Goldenring JM CE. Getting into adolescent heads: An Essential Update. Contemporary Pediatrics 2004; 21:64 https://peds.arizona.edu/sites/default/files/curriculum-files/headss.pdf

11.Statacorp. Stata Statistical Software: release 14.: TX: StataCorp LP; 2015. 
12.Transplant NBa. Organ Donation and Transplantation Activity Report.

https://nhsbtdbeblobcorewindowsnet/umbraco-assets-corp/16537/organ-donation-and-transplantationactivity-report-2018-2019pdf. 2019.

13.Masding MG KR, MacHugh B, Gale GD, Brown MB, McAulay A. Non-attendance at a diabetes transitional clinic and glycaemic control. Practical Diabetes International. 2010;27(3):109-10.

14.Watson AR. Non-compliance and transfer from paediatric to adult transplant unit. Pediatr Nephrol. 2000;14(6):469-72.

15.Andreoni KA, Forbes R, Andreoni RM, Phillips G, Stewart H, Ferris M. Age-related kidney transplant outcomes: health disparities amplified in adolescence. JAMA Intern Med. 2013;173(16):1524-32.

16.Van Arendonk KJ, Boyarsky BJ, Orandi BJ, James NT, Smith JM, Colombani PM, et al. National trends over 25 years in pediatric kidney transplant outcomes. Pediatrics. 2014;133(4):594-601.

17.Dobbels F, Ruppar T, De Geest S, Decorte A, Van Damme-Lombaerts R, Fine RN. Adherence to the immunosuppressive regimen in pediatric kidney transplant recipients: a systematic review. Pediatr Transplant. 2010;14(5):603-13.

18.Bhaumik S WJ, Barrett M, Raju B, Burton T, Forte J. Transition for Teenagers With Intellectual Disability: Carers' Perspectives. Journal of Policy and Practice in Intellectual Disabilities. 2011;8(1):5361.

19.Audit NOfC. GDPR GUIDANCE FOR CLINICAL AUDIT MAY 2019. https://s3-eu-west1.amazonaws.com/noca-uploads/general/NOCA_GDPR_Guidance_for_Clinical_Audit_May_2019.pdf

\section{Tables}

Table 1: Transplant group comparison for demographics and outcomes

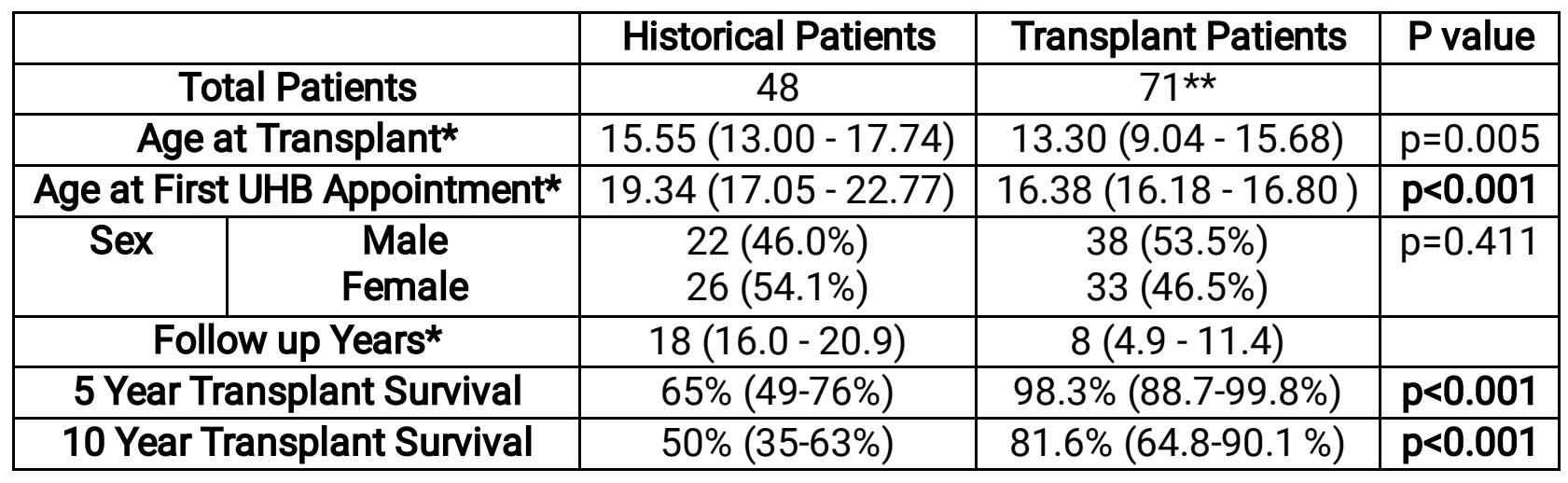

* median interquartile range

Table 2: Questionnaire outcomes for service evaluation 


\begin{tabular}{|l|l|l|}
\hline $\begin{array}{l}\text { Question } \\
\text { Has the Young Adult clinic... }\end{array}$ & Mean & Std Deviation \\
\hline Increased your understanding of your medical condition? & 5.56 & 1.37 \\
\hline Increased your confidence in managing your medical condition? & 5.62 & 1.43 \\
\hline Increased your confidence in managing your medicines? & 5.51 & 1.34 \\
\hline Made you feel more prepared for an adult kidney clinic? & 5.74 & 1.46 \\
\hline Helped you plan for the future? & 4.97 & 1.56 \\
\hline $\begin{array}{l}\text { If you had a kidney related problem would you feel comfortable contacting the young adult service? } \\
\text { If you had a more personal issue, how comfortable would you be in discussing it with a member of } \\
\text { team? }\end{array}$ & 5.63 & 1.82 \\
\hline
\end{tabular}

